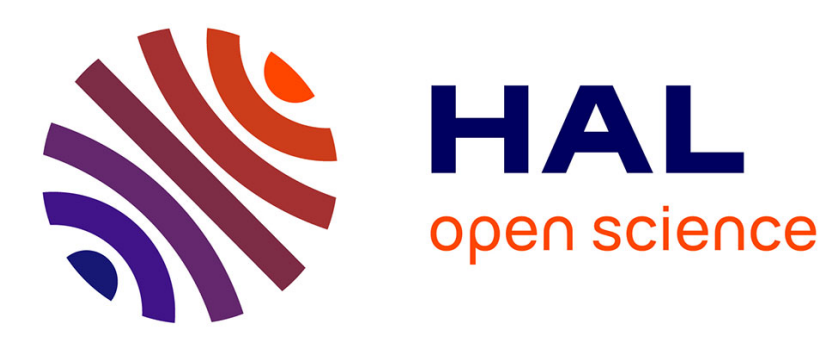

\title{
Approche métapsychologique de la notion de Réseau et enjeux actuels dans la clinique des groupes

\author{
Clarisse Vollon
}

\section{To cite this version:}

Clarisse Vollon. Approche métapsychologique de la notion de Réseau et enjeux actuels dans la clinique des groupes. Revue de psychothérapie Psychanalytique de Groupes, 2019, 73, pp.61-69. 10.3917/rppg.073.0061 . hal-02485556

\section{HAL Id: hal-02485556 https://hal-amu.archives-ouvertes.fr/hal-02485556}

Submitted on 20 Feb 2020

HAL is a multi-disciplinary open access archive for the deposit and dissemination of scientific research documents, whether they are published or not. The documents may come from teaching and research institutions in France or abroad, or from public or private research centers.
L'archive ouverte pluridisciplinaire $\mathbf{H A L}$, est destinée au dépôt et à la diffusion de documents scientifiques de niveau recherche, publiés ou non, émanant des établissements d'enseignement et de recherche français ou étrangers, des laboratoires publics ou privés. 


\section{APPROCHE MÉTAPSYCHOLOGIQUE DE LA NOTION DE RÉSEAU ET ENJEUX ACTUELS DANS LA CLINIQUE DES GROUPES}

\section{CLARISSE VOLLON}

Si l'étymologie du mot réseau désigne un filet pour capturer, pour retenir, ou encore un ensemble de lignes entrelacées, elle recouvre un sens spécifique dans l'analyse des groupes. En effet, Foulkes propose dès les années 1950 un nouveau modèle du groupe où la notion de réseau, The Network dans sa version originale, a une place théoricoclinique centrale : elle lui permet de penser et définir la forme et l'organisation groupales, les modalités de communication entre ses membres, la place de chacun des individus dans le groupe. Comment la notion de réseau a-t-elle évolué ces dernières années dans la littérature scientifique nationale et internationale de l'analyse de groupe ? Quelles sont les articulations possibles entre cette notion et les autres modèles psychanalytiques du groupe ? En quoi peut-il être pertinent d'avoir recours à la notion de réseau pour construire et mener nos pratiques de groupe actuelles ? Pour répondre à ces questions, je propose dans cet article de revenir sur la notion de réseau telle qu'elle a été pensée par Foulkes, dans l'ensemble de son œuvre et par ses successeurs mais aussi de discuter et d'articuler cette notion avec le modèle de l'appareil psychique groupal (Kaës, 1976, 2015). Ce travail d'articulation et son illustration clinique à partir d'une séance de supervision permettront, notamment, d'apporter un éclairage supplémentaire sur les modalités de liaisons durant les tout premiers temps de la formation d'un groupe, et plus largement les dynamiques en jeux dans son espace intersubjectif.

\section{LA NOTION DE RÉSEAU}

Foulkes était un psychiatre, neurologue et psychanalyste allemand, qui a immigré à Londres en 1933 sur invitation d'Ernest Jones pour fuir le régime nazi. Après plus de onze ans d'expérience dans les dispositifs analytiques individuels, il commence, en 1941, à travailler avec 
des groupes au sein de l'hôpital militaire de Northfield (Hinshelwood, 2009). Rapidement, il propose une modélisation des dynamiques groupales qu'il peut observer dans le service où il intervient. Les principes de ce modèle sont exposés pour la première fois dans un court article de 1946, "On group Analysis ». Ce modèle s'étaye sur trois influences : les travaux de Kurt Goldstein (1952) qui envisagent l'organisation neurobiologique comme l'équivalent d'une Gestalt - l'organisme est un tout dont les parties ne peuvent être comprises que dans un contexte plus global -, la psychanalyse et la socioanalyse avec les travaux de Karl Mannheim (1943) qui envisage l'étude du groupe comme porte d'accès pour comprendre des dynamiques groupales plus élargies et plus complexes. Foulkes dégage alors les prémices d'une approche du groupe comme un tout. Pour autant, il faut attendre 1957 pour qu'il développe le modèle de la matrice et plus spécifiquement celle de réseaux.

Ainsi, selon Foulkes, le groupe comme matrice est un ensemble de réseaux exactement comme le cerveau est un ensemble de réseaux de fibres et de cellules qui forment une unité complexe. Ces réseaux, transpersonnels, sont la base d'action de tous les processus psychiques dans le groupe de la même façon que «l'esprit » d'une personne est la base d'action de tous ses processus mentaux. Ici, l'individu est envisagé comme un point nodal de ces réseaux, comme suspendu en eux. Plus spécifiquement, les membres d'un groupe sont « dans un état d'interaction dans un champ commun en interpénétration et communication. Ils parlent tantôt par une bouche, tantôt par une autre ${ }^{1} »$. C'est pour cette raison que Foulkes utilise également le terme de « réseaux de communication ». Un peu plus tard, il revient sur son modèle et propose de dégager une véritable psychopathologie des réseaux. Ainsi, les patients feraient partie intégrante de tout un réseau d'individus en interaction, dont ils ne seraient que des victimes ou des boucs émissaires, sinon des symptômes ${ }^{2}$. Par extension, chaque organisation pathologique correspondrait à une organisation de réseaux spécifiques. Toutefois, Foulkes précise que ces réseaux sont à envisager comme une organisation psychique globale où l'inconscient peut se manifester sous diverses formes, et non comme un système d'interactions sociales superposées dans lesquelles des esprits individuels interagissent les uns avec les autres. Cette nuance nous permet de faire un distinguo entre la notion de réseaux de Foulkes et celle actuellement utilisée par les systémiciens.

Pour conclure, la notion de réseau chez Foulkes peut être envisagée de façon quadridimensionnelle. Tout d'abord comme le maillage structurel d'un groupe, car le réseau propose une topographie du groupe.

1. S.H. Foulkes, J. Anthony, Psychothérapie de groupe, approche analytique, Paris, Epi, 1957, p. 326.

2. S.H. Foulkes, Introduction to Group-Analytic Psychotherapy: Studies on the Social Integration of Individuals and Groups, London, Karnac Books, p. 225. 
Ensuite, le réseau peut s'envisager comme le lieu d'émergence des processus psychiques individuels et groupaux. Il est également le champ d'interactions des membres d'un groupe, et constitue enfin l'ensemble des canaux de communication entre les membres d'un groupe. Comme le remarque Pines (1989) et Hinshelwood (2009), les changements apportés à ce modèle ont été peu nombreux jusqu'à aujourd'hui. Pourtant, l'utilisation de la neurobiologie pour comprendre le fonctionnement groupal s'est développée ces dernières années. Ainsi, Kinley (2016) a proposé un modèle relationnel dynamique, qui suggère une compréhension des processus neurobiologiques impliqués dans le développement du groupe. Les phases du développement du groupe observé reflètent, selon cet auteur, une réorganisation sous-jacente des circuits neuronaux lorsque les membres du groupe progressent dans leur traitement en groupe. Seulement, la notion de réseau est envisagée dans ces travaux exclusivement dans sa dimension neurobiologique. Or Foulkes n'a pas utilisé la neurobiologie pour expliquer le phénomène groupal mais s'est plutôt inspiré de la neurobiologie pour construire son propre modèle du groupe et en penser la forme et la dynamique. C'est dans cette perspective que je propose d'articuler la notion de réseau avec celle d'appareil psychique groupal (Kaës, 1976, 2015).

\section{LES RÉSEAUX, UNE MÉTAPHORE DE L'ESPACE DU LIEN}

Kaës $(1976,2015)$ définit l'appareil psychique groupal comme une structure indépendante des psychés qui « assemble en un arrangement combinatoire selon ses lois propres, mais qui est aussi une construction intériorisée par les membres du groupe ${ }^{3} »$. Trois espaces constituent l'Appareil psychique groupal : l'espace du sujet, l'espace du lien et l'espace du groupe. Je vais m'arrêter ici plus spécifiquement sur l'espace du lien, appelé aussi l'espace intersubjectif.

L'espace intersubjectif est l'espace où les liens entre les membres du groupe se développent, se stabilisent, mais aussi se rompent dans une dynamique psychique qui leur est propre. Ces liens présentent des caractéristiques similaires à celles des réseaux. En effet, les liens, tout comme les réseaux, accueillent un ensemble de processus psychiques, ils sont l'expression des mouvements plus ou moins stables des investissements, des représentations et des actions qui associent plusieurs sujets pour accomplir certaines réalisations psychiques qu'ils ne pourraient pas obtenir seuls ${ }^{4}$. Tout comme les réseaux, les liens ont également une logique propre qui va soutenir le champ d'interaction des membres du groupe. Enfin, les liens, comme les réseaux, peuvent être appréhendés dans une dimension topographique, ils constituent un espace de réalité psychique spécifique construit à partir de la matière

3. R. Kaës, L'extension de la psychanalyse, Paris, Dunod, 2015, p. 126.

4. Ibid., p. 86. 
psychique engagée dans les relations entre deux ou plus de deux sujets ${ }^{5}$. Toutefois, contrairement aux réseaux, les liens qui structurent l'espace intersubjectif ne constituent pas à eux seuls la structure du groupe et les liens ne sont pas appréhendés comme des canaux de communication. Malgré tout, la notion de réseaux peut être éclairante pour comprendre l'organisation de cet espace intersubjectif. En effet, elle peut être envisagée comme une modélisation des investissements psychiques, des alliances inconscientes, des pactes dénégatifs, des chemins empruntés par les pulsions. Dans cette perspective, la notion de réseau pourrait constituer une métaphore efficace de l'espace du lien, nous permettant d'envisager l'espace intersubjectif comme un espace câblé, un maillage à la qualité plastique à l'instar de la plasticité neuronale, qui parfois se renforce sous l'effet des alliances inconscientes, parfois se rompt sous l'effet de séparations ou de trauma. L'enveloppe psychique constituerait non seulement la bordure des espaces intra-, inter- et transsubjectif mais aussi la gaine de myéline de ces réseaux de liens (Vollon, Gimenez, 2015).

Dans l'illustration clinique qui va suivre je vais utiliser cette métaphore pour comprendre la dynamique d'un groupe de professionnels que je supervise.

\section{ILLUSTRATION CLINIQUE : DE L'HYPEREXCITABILITÉ DES RÉSEAUX À LEUR RÉORGANISATION DANS L'ESPACE DU LIEN}

J'ai été sollicitée par une mission locale ${ }^{6}$ du sud de la France pour intervenir auprès de ses salariés afin qu'ils puissent bénéficier d'un espace de paroles et de réflexions autour de la population qu'ils accompagnent ainsi que de leurs modalités de travail. Suite aux entretiens préliminaires effectués avec l'équipe et la direction, j'ai proposé un dispositif de supervision par le psychodrame, mensuel et ouvert à tous. Durant ces séances, je proposais aux salariés d'utiliser l'association libre et le jeu pour favoriser une mise en mots parfois difficile sur des situations concrètes du quotidien professionnel, que ce soit avec les jeunes qu'ils accueillent ou avec leurs collègues. Le cadre des séances était construit ainsi : les salariés étaient invités, dans un premier temps, à présenter des situations professionnelles qui leur posaient problème. Dans un second temps, nous utilisions ces situations comme supports pour élaborer des scénarios de jeux afin d'étayer notre réflexion. Les règles de confidentialité, de régularité, de ponctualité, du respect

5. Ibid., p. 85.

6. Les missions locales ont été créées dans les années 1980 en France pour favoriser l'insertion professionnelle et sociale des jeunes âgés de 16 à 25 ans. Leurs domaines d'intervention sont variés : emploi, formation, logement, santé, mobilité... Même si une mission locale s'adresse en priorité aux jeunes qui sont sortis du système scolaire ou qui n'ont pas d'emploi, elle propose aussi un accompagnement à ceux qui poursuivent des études ou sont actifs. Organisées en réseau, elles sont réparties sur tout le territoire et proposent de nombreux points d'accueil. 
de la parole et de la bienveillance soutenaient nos échanges. Dans l'illustration qui va suivre, les noms des participants ainsi que l'ensemble des éléments qui permettraient de reconnaître les institutions et les usagers ont été modifiés.

\section{L'hyperexcitabilité des réseaux durant la mise en groupe}

Je vais présenter la première séance de cette supervision. Les participants sont Juliette, Sarah, Olivia, Maria, Marion, Fanny, Aurélie, Pauline, Muriel, Solenne et Paul. Lorsque nous nous installons, il manque une personne, Muriel, qui est la cadre coordinatrice dans la mission locale, nous ne connaissons pas les raisons de son absence et sa place restera vide durant toute cette première séance.

Paul prend rapidement la parole : "Voilà, j'ai eu un problème, il n'y a pas très longtemps avec un jeune, Younes. On sortait d'un groupe de parole avec d'autres jeunes et, d'un coup, il s'est mis à s'énerver. Je crois qu'il a carrément pété les plombs, il a menacé de se tuer et nous avec. C'était limite délirant, on a dû faire partir les autres jeunes et isoler Younes pour qu'il se calme. » Je questionne alors sur les raisons de cette décompensation, Paul précise : « On lui a dit qu'il n'avait plus le droit aux prestations sociales. » Puis il continue : «Le lendemain, il est revenu à la mission locale, c'était l'heure de ma pause, je n'ai pas voulu le recevoir dans le bureau, pour le principe. Mais, du coup, c'était compliqué. Il voulait appeler son père en Martinique pour qu'on lui envoie des sous, j'avais peur qu'il pète encore un plomb, à côté les autres sont rentrés dans le bureau pour ne pas rester avec nous dans le couloir. » Pendant que Paul parle, les autres membres du groupe interviennent continuellement dans un brouhaha diffus. Ces interventions sont de plusieurs registres : informatives sur le jeune, demandes d'éclaircissements ou de précisions sur la situation. Je suis obligée d'intervenir à plusieurs reprises pour demander au groupe de laisser Paul terminer de nous présenter la situation qui lui a posé problème. Je repère alors que la chaîne associative groupale est particulièrement dense, chargée d'interventions multiples qui transgressent la règle du respect de la parole. Mon esprit est saturé par ce flot continu de paroles et je ressens une légère nausée. Finalement, Paul parvient à conclure : « En fait, je me demande comment j'aurai dû faire pour éviter qu'il pète un plomb comme ça, mais aussi pour protéger les autres. » Éviter que quelqu'un décompense, protéger les autres, se protéger soi-même, les mots de Paul résonnent en moi comme une demande groupale de se protéger de quelque chose.

Nous pouvons repérer dans ces tout premiers temps de la vie du groupe, une saturation de la chaîne associative groupale soutenue par une hyperexcitabilité des réseaux de communication. En effet, les membres du groupe interagissent de façon continue durant ce premier temps d'échanges dans l'espace intersubjectif : chacune des paroles de 
Paul fait l'objet d'interventions, d'associations, de commentaires de la part des autres participants. Tout semble se passer comme s'il n'était pas possible pour le groupe de laisser la parole se déposer sans qu'elle ne génère une réaction en chaîne incontrôlable au sein des réseaux de l'espace du lien. Comment expliquer cette hyperexcitabilité ? Le moment de la mise en groupe est, comme chacun sait, une période sensible dans une dynamique groupale. Entre l'augmentation de la porosité des enveloppes psychiques (Vollon, Gimenez, 2014) et l'émergence d'angoisses massives, le moment de mise en groupe peut générer des mouvements maniaques défensifs importants. Ajouté à cela, il est possible que l'absence non expliquée de la cadre coordinatrice soit venue entraver ce processus de mise en groupe en générant chez les participants un sentiment de grande vulnérabilité, voire un sentiment d'abandon. Plus spécifiquement, il est possible que cette absence soit venue provoquée une crainte du côté des participants que les échanges initiés en groupe conduisent peut-être à les rendre fous, à s'effondrer, à s'agresser les uns les autres... Voire à me faire moi-même partir en tant que conducteur du groupe, m'absentant alors comme la coordinatrice.

L'hyperexcitabilité des réseaux de communication dans l'espace du lien pourrait ainsi être envisagée comme l'expression de ces multiples processus qui s'accumulent et se bousculent durant ce début de vie groupale. Dans cette perspective, mon éprouvé corporel contretransférentiel peut être entendu comme une réaction défensive à ce trop-plein d'évènements psychiques qui traversent le groupe.

\section{Réorganisation des réseaux et figuration de cette réorganisation par le jeu}

Pour temporiser cette hyperexcitabilité, je décide de formuler une intervention contenante et apaisante qui utilise le cadre de la supervision pour renforcer la gaine de myéline qui borde le câblage de ces réseaux mais qui permet aussi de mettre en lumière les angoisses qui traversent le groupe. Dit autrement, je tente donc de faire une intervention biface sur la forme et sur le fond de ce groupe comme un tout, me positionnant comme garante du cadre de travail et je dis : « Cette supervision et les règles qui l'organisent sont une nouvelle façon pour vous d'être les uns avec les autres, une nouvelle façon de fonctionner qui change de vos habitudes de travail. Et puis aussi l'absence de Muriel peut être inquiétante, mais nous pouvons réfléchir et élaborer avec bienveillance, même si certains d'entre nous sont absents. » Le groupe reste silencieux, puis Fanny prend la parole : «En ce moment, je suis un jeune fiché $S$, il a fait de la prison, il est suivi par le $\operatorname{SPIP}^{7}$ et maintenant il cherche du travail. Tout le monde est inquiet. » Je lui demande de nous en dire un peu plus sur ce jeune : «Il a 21 ans, il est grand,

7. Service pénitentiaire d'insertion et de probation. 
massif, impressionnant, on ne sait pas qui est sa famille, il est tout seul, en fait. Pendant le premier rendez-vous, il refuse de me serrer la main parce que je suis une femme, il me tend le coude. Un peu avant, il est parti pendant un mois sans que personne ne sache où, tout le monde pense à la Syrie. Pendant l'entretien, il est dans de multiples demandes, et on a l'impression qu'en face on n'existe pas. Pour la première fois, j'ai débordé mon cadre, je l'ai reçu pendant plus d'une heure, ce qui m'a fait partir tard du travail. J'ai pris contact avec tout le monde ensuite, le SPIP, son assistante sociale. J'ai aussi contacté l'association qui l'héberge, mais ils n'ont pas été très coopératifs. Alors j'ai envoyé un mail à la directrice de cette association pour lui faire part de mon inquiétude et regretter l'absence d'échanges avec son équipe. La directrice a très mal pris mon mail et Muriel aussi, du coup, elle veut qu'on se rencontre tous avec le jeune, mais moi je ne pense pas que ce soit la place de ce jeune, on doit d'abord se rencontrer nous, je ne sais pas quoi faire. » Pendant qu'elle présente sa situation, le groupe est, cette fois, attentif. Juliette intervient en soulignant la solitude qu'elle peut rencontrer dans sa pratique. Je pense alors à l'absence de cette cadre coordinatrice. Je me dis que l'absence et le manque commencent à pétrir l'ébauche d'une illusion groupale : l'image d'un groupe orphelin me vient. Je propose aux participants de jouer la scène de cette première rencontre entre Fanny et ce jeune. Fanny souhaite jouer le rôle de ce jeune, Marion accepte de jouer le rôle de Fanny. Je propose de modifier les identités. Fanny sera Abdel, 18 ans, Marion décide de s'appeler Olga et de lui donner l'âge de 45 ans. Durant le jeu, Abdel est nonchalant et dans de multiples demandes. Il demande des sous pour s'acheter des vêtements, Olga lui répond : " On va voir si on peut débloquer des sous mais, vous savez, ça n'est pas le but de la mission locale. Ici, on est là pour vous aider à trouver du travail et après vous pourrez vous acheter de nouveaux vêtements. On va commencer par votre CV. » Il lui rétorque : "Mais, moi, je veux des vêtements. Vous, vous en avez bien. Ben, moi aussi, je veux des nouveaux vêtements. » Olga commence à perdre patience : " Oui, mais moi je travaille pour me les acheter, ça vient pas comme ça en un claquement de doigts. » L'entretien se termine et les jeunes femmes retournent à leur place dans le groupe. Durant ce jeu, je ressens de l'exaspération mais aussi de l'agressivité envers Abdel, des ressentis qui circuleront également lors des échanges entre les membres du groupe autour de la scène : tout le monde s'accordera à dire qu'Abdel était vraiment insupportable et que beaucoup sont « comme ça », dans des demandes immédiates qui obligent les salariés parfois à sortir de leur rôle et à devoir faire des accompagnements « pour un peu tout et n'importe quoi ». L'image me vient d'oisillons dans un nid qui ouvriraient leurs becs pour attendre leur repas.

Nous pouvons repérer que, durant cette seconde partie de mise en groupe, les réseaux se réorganisent : l'hyperexcitabilité a laissé la 
place à une circulation apaisée de la communication. Les vécus groupaux d'abandon et de vulnérabilité parviennent alors à se scénariser à travers la situation rapportée par Fanny qui est débordée par ce jeune qu'elle reçoit en entretien mais aussi les propos de Juliette qui se sent seule dans sa pratique professionnelle. Cette réorganisation trouve alors une figuration dans le jeu : Abdel, dans ses multiples demandes peut être envisagé comme une figuration de l'hyperexcitabilité des réseaux de communication et plus globalement de ce groupe orphelin de sa cadre coordinatrice qui sature l'espace de paroles en interventionsdemandes multiples. Olga figurerait alors le cadre-gaine de myéline que j'ai tenté de ramener par mon intervention, et qui viendrait border et contenir ces demandes multiples.

\section{EN GUISE DE CONCLUSION}

J'ai proposé, dans cet article, d'appréhender la notion de réseau dans une approche métapsychologique et de revenir sur ses spécificités dans l'analyse de groupe. En articulant la notion de réseau avec le modèle de l'appareil psychique groupal de Kaës, il est possible de dégager une métaphore de l'espace psychique du lien, cet espace qui peut parfois être difficile à appréhender compte tenu de la complexité des processus qui le traversent et plus largement de son organisation. De plus, en utilisant cette métaphore pour penser la dynamique d'un groupe de supervision il est possible de constater que les réseaux de l'espace intersubjectif sont dynamiques, pouvant alterner entre une hyperexcitabilité et une réorganisation stable et scénarisable. Plus largement, revenir sur la notion de réseau dans l'œuvre de Foulkes permet d'apprécier l'importance de l'approche pluridisciplinaire dans la construction des modèles psychanalytiques du groupe : en effet, c'est l'articulation de la neurobiologie, de la socioanalyse et de la psychanalyse qui a permis, dans les années 1950, la construction du modèle du groupe comme matrice. Cette pluridisciplinarité est encore à promouvoir aujourd'hui et peut trouver sa pertinence pour nous éclairer sur les dynamiques de groupes actuelles.

\section{BIBLIOGRAPHIE}

BION, W.R. 1961. Recherche sur les petits groupes, Paris, Puf.

FOULKES, S.H. 1946. «On group analysis », International Journal of Psycho-Analysis, 27, p. 46-51.

Foulkes, S.H. ; Anthony, J. 1957. Psychothérapie de groupe, approche analytique, Paris, Epi.

Foulkes, S.H. 1983. Introduction to Group-Analytic Psychotherapy: Studies on the Social Integration of Individuals and Groups, London, Karnac Books.

Foulkes, S.H. 1990. Selected Papers of S.H. Foulkes: Psychoanalysis and Group Analysis, London, Karnac Books. 
GoldsteIn, K. 1952. La structure de l'organisme, Paris, Gallimard.

Hinshelwood, R.D. 2009. «Bion et Foulkes, le groupe comme un tout », Revue de psychothérapie psychanalytique de groupe, $\mathrm{n}^{\circ}$ 52, p. 99-109.

KAËs, R. 1976. L'appareil psychique groupal, Paris, Dunod.

KAËs, R. 2015. L'extension de la psychanalyse, Paris, Dunod.

KinLEY, J.L. ; ReYNo, S. 2016. « Dynamic relational group psychotherapy: a neurobiologically informed model of change », International journal of groupe psychotherapy, 66, p. 161-178.

Vollon, C. ; Gimenez, G. ; Bonnet, C. 2015a. « Mise en groupe, psychose et porosité des enveloppes psychiques », Revue de psychothérapie psychanalytique de groupe, $\mathrm{n}^{\circ} 64$, p. 203-214.

Vollon, C. ; Gimenez, G. ; Bonnet, C. 2015b. « The notion of the Matrix: new perspectives in group psychotherapy for psychotic patient », Group Analysis, 48, p. 174-186. 